${ }^{1}$ Escuela de Psicología. Pontificia Universidad Católica de Chile.

${ }^{2}$ Universidad de La Serena. La Serena, Chile.

${ }^{3}$ Hospital del Trabajador. Asociación Chilena de Seguridad. Santiago de Chile. apsicóloga. PhD.

bPsicóloga. Estudiante Programa de Doctorado en Psicología PUC.

Fuente de apoyo financiero: Proyecto VRI-PUC a E. Guic.

Recibido el 6 de agosto de 2013, aceptado el 11 de junio de 2014

Correspondencia a: Eliana Guic Av. Vicuña Mackenna 4860, Macul, Santiago. eguic@uc.cl

\section{Adaptación cultural y validación de la versión chilena del Cuestionario de Discapacidad Roland-Morris}

\author{
ELIANA GUIC ${ }^{1, \mathrm{a}}$, SUSAN GALDAMES ${ }^{2, \mathrm{~b}}$, POLICARPO REBOLLEDO $^{3}$
}

\section{Validation and cultural adaptation of the Chilean version of the Roland-Morris Disability Questionnaire}

Background: The evaluation of disability is highly relevant for the study and clinical follow-up of pain. Reliable and culturally valid instruments are required for this purpose. Aim: To adapt and to validate the Roland-Morris Disability Questionnaire (RDQ) to measure disability in low back pain Chilean patients and to study the psychometric properties of an abbreviated six item version of this instrument. Material and Methods: The instrument's original version was translated and back translated into Spanish, and specialists ensured the cultural validation to Chile. Disability mental health using the Goldberg General Health Questionnaire (GHQ-12) and pain using a visual analogue scale (VAS) were evaluated in 206 patients, during a medical appointment due to acute low back pain. Results: The Chilean version of the original 24-item RDQ and the new six item version showed adequate internal consistency. The short version also showed a good convergent validity. It had the same pattern of correlations with VAS and GHQ-12 scales, as the original 24 item scale. Greater disability was associated with higher pain intensity and poorer mental health. The construct validity analysis identified one factor, for both versions of the RDQ. Conclusions: The adapted Chilean version of the original $R D Q$ is a reliable and valid questionnaire, as well as the new abbreviated six items version, which showed adequate psychometric properties.

(Rev Med Chile 2014; 142: 716-722)

Key words: Disability, evaluation; Low back paint; Questionnaires; Reproducibility of results.
41

1 dolor músculo-esquelético más frecuente en la población adulta chilena es el dolor de espalda (ENS 2009) ${ }^{1}$. El 48,2\% padeció dolor de espalda y $29,8 \%$ sintió el dolor en la parte baja de la espalda, la semana anterior a la encuesta. La mayoría de estas personas reporta que padece este dolor en forma crónica y es evaluado como el segundo dolor músculo-esquelético más intenso. Cifras similares se habían observado en la II Encuesta de Calidad de Vida y Salud realizada el $2006^{2}$, donde se estimó que $44 \%$ de los chilenos mayores de 15 años había padecido dolor de espalda o lumbago durante las dos semanas previas a la encuesta. Estas cifras muestran que el dolor lumbar es un problema que afecta a un grupo significativo de chilenos, probablemente perjudicando su vida personal y laboral, así como sucede en otros países desarrollados o en vías de desarrollo ${ }^{3}$.

El Cuestionario Roland-Morris (RDQ) evalúa discapacidad física y psicológica debida al dolor lumbar o el impacto de este dolor en la vida de 
los pacientes. La discapacidad es un indicador del bienestar personal, cuya medición es útil tanto para la investigación como para el monitoreo clínico de pacientes con dolor lumbar. El RDQ es un instrumento de 24 ítems, de amplio uso a nivel internacional, por lo que ha sido traducido y adaptado a diversas culturas. Estas validaciones han mostrado buenos indicadores psicométricos, por lo que se cuenta con un instrumento adecuado para evaluar discapacidad por dolor lumbar en diferentes países. Citamos por ejemplo la versión alemana $^{4}$, tunesina ${ }^{5}$, española ${ }^{6}$, brasilera ${ }^{7}$, chi$\mathrm{na}^{8}$, gujarati (India) ${ }^{9}$, japonesa $\mathrm{a}^{10} \mathrm{y}_{\text {coreana }}{ }^{11}$. Sin embargo, carecemos de una versión validada en Chile, que nos permita usar con propiedad este instrumento en nuestro país.

El objetivo de este trabajo fue adaptar y validar el RDQ original de 24 ítems, en pacientes chilenos que consultan por dolor lumbar. Además, nuestro trabajo estudia las características psicométricas preliminares de una nueva versión abreviada, de 6 ítems, la cual facilitaría el monitoreo de la discapacidad, especialmente en la práctica clínica o para seguimientos telefónicos. Se han hecho esfuerzos previos de obtener versiones más breves de este cuestionario, de 18 y 11 ítems, las que han demostrado adecuadas características psicométricas ${ }^{12,13}$.

\section{Materiales y Método}

\section{Sujetos}

El cuestionario fue aplicado a una muestra intencional de 206 empleados, que consultaron consecutivamente por dolor lumbar agudo -una semana o menos de dolor- en el Servicio de Urgencia del Hospital del Trabajador en Santiago de Chile. La gran mayoría de los participantes sujetos a la Ley 16.744, aunque no se descartó a pacientes con dolor lumbar agudo de origen común, no laboral. Fueron invitados a participar los pacientes que consultaron por primera vez o por un nuevo episodio de dolor lumbar y que estuvieran trabajando en ese momento. Los criterios de exclusión fueron haber sido sometido a cirugía durante los tres meses previos a la investigación; embarazo y haber consultado por dolor lumbar durante el último año.

\section{Instrumentos}

El Cuestionario Roland-Morris para medir discapacidad es un instrumento auto-administrado que se construyó a partir del Sickness Impact Profile (SIP), construido para evaluar personas con dolor lumbar ${ }^{14}$. El RDQ consta de 24 ítems, con dos opciones de respuesta (sí - no) y evalúa la discapacidad física y psicológica, en el funcionamiento diario, relacionada con la enfermedad. Da un puntaje total que fluctúa entre 0 y 24 , el cual se obtiene sumando las respuestas "Sî" ( $\mathrm{Sí}=$ 1 punto; No = 0) del total de ítems administrados. A mayor puntaje mayor discapacidad asociada al dolor lumbar.

En el presente estudio se usó la versión completa del instrumento de 24 ítems, y además una versión abreviada de 6 ítems. Ver Anexo 1. Estos ítems se seleccionaron del instrumento original siguiendo los siguientes criterios: Mantener una representatividad de las actividades evaluadas en el instrumento original; Seleccionar las actividades más frecuentes de la vida cotidiana para pesquisar discapacidad, incluso en pacientes agudos, que normalmente consultan a horas o pocos días desde que comienza el dolor y la discapacidad; se consideró los ítems usados en una versión previa de 11 ítems ${ }^{12}$.

También se utilizó la Escala Visual Análoga (EVA) para evaluar la intensidad del dolor ${ }^{15} \mathrm{y}$ el Cuestionario de Salud General de Goldberg $\left(\right.$ GHQ-12) ${ }^{16}$, adaptado en Chile ${ }^{17}$, el cual evalúa salud mental general, más específicamente, vulnerabilidad a presentar cuadros de ansiedad y depresión. También se evaluó otras variables relevantes como Índice de Masa Corporal (IMC).

\section{Procedimiento}

$\mathrm{La}$ adaptación del instrumento original se realizó mediante una traducción inicial del cuestionario del idioma inglés al español, realizada por traductores bilingües. Posteriormente, se realizó una retro-traducción, revisada por especialistas en el tema, quienes velaron por la adaptación cultural, la conservación de la versión original y la comprensibilidad de todos los ítems que componen el cuestionario en versión preliminar. Esta versión preliminar, además fue testeada con una muestra piloto antes de la aplicación del instrumento a la muestra total. En el piloto se observó una gran desigualdad en la capacidad lectora de los pacientes, por lo que se optó por realizar una aplicación asistida, donde el instrumento se lee a los pacientes.

Los pacientes que aceptaron participar en el 
estudio firmaron un consentimiento informado y todos los procedimientos estuvieron de acuerdo a las recomendaciones éticas de la Escuela de Psicología de la Pontificia Universidad Católica de Chile y del Comité de Ética del Hospital del Trabajador de la Asociación Chilena de Seguridad. Todos los instrumentos fueron aplicados al momento de la consulta inicial por dolor agudo, en la sala de espera.

\section{Análisis}

Los análisis estadísticos fueron realizados mediante el programa estadístico SPSS.18. Para estimar la confiabilidad y validez del instrumento se utilizaron varios procedimientos. Primero, se calculó la consistencia interna de los instrumentos utilizando el Alfa de Cronbach para la versión de 24 ítems y para la versión corta, de 6 ítems.

Para examinar la validez convergente se correlacionaron los puntajes totales de ambas versiones del RDQ, con la intensidad del dolor (EVA); salud mental general (GH12); índice de masa corporal y sexo. La validez de constructo fue analizada llevando a cabo un análisis de Maximun Likehood y Rotación Oblimin, tanto para el instrumento original de 24 ítems, así como para el instrumento de 6 ítems. Previo a esto, se observó que las variables eran apropiadas para el procedimiento escogido.

\section{Resultados}

\section{Descripción de la muestra}

La edad promedio de los sujetos fue de 36,8 años (18-64 años); 75\% hombres y 25\% mujeres. El 67\% de la muestra reportó no haber consultado previamente por dolor lumbar. La mayor parte del restante 33\% había consultado previamente sólo en una oportunidad, pero nadie durante el año previo a la consulta actual. El $22 \%$ de la muestra reportó realizar trabajos pesados en forma cotidiana (Tabla 1).

\section{Consistencia interna}

El Alfa de Cronbach del RDQ fue de 0,81 y para la versión de 6 ítems fue de 0,78 , por lo tanto, una consistencia interna adecuada para ambas versiones del instrumento (Tabla 2).

\section{Validez convergente}

Los puntajes de ambas versiones están altamente correlacionados $(\mathrm{r}=0,81 ; \mathrm{p}<0,000)$. Para estudiar la validez convergente, se correlacionó a otras variables del estudio con ambas versiones, de 24 y 6 ítems. Las dos versiones del RDQ tuvieron en general el mismo patrón de correlaciones con estas variables. Ambas versiones se correlacionaron positivamente con peor salud mental o mayor

Tabla 1. Descripción de la muestra

\begin{tabular}{|lccc|}
\hline & Promedio & Desviación típica & Puntajes Min.-Max. \\
\hline Edad & 36,80 & 9,93 & $18,00-64,00$ \\
Índice masa corporal & 26,15 & 3,82 & $16,67-38,20$ \\
Años de antigüedad laboral & 6,324 & 6,65 & $0,16-30$ \\
\hline
\end{tabular}

Tabla 2. Resultados descriptivos. Puntajes observados en los instrumentos utilizados $(\mathbf{n}=\mathbf{2 0 6})$

\begin{tabular}{|llccc|}
\hline Instrumento & & Promedio & Desviación típica & Puntajes Min.-Max. \\
\hline EVA* & (Dolor) & 7,14 & 1,95 & $3-10$ \\
RDQ** & (Discapacidad) & 15,91 & 4,50 & $3-24$ \\
RDQ-6*** & (Discapacidad) & 4,42 & 1,33 & $0-6$ \\
GHQ-12**** & (Salud mental) & 3,22 & 2,86 & $0-12$ \\
\hline
\end{tabular}

*Escala Visual Análoga; **Cuestionario Roland-Morris; ${ }^{* * * C u e s t i o n a r i o ~ R o l a n d-M o r r i s-6 ~ i ́ t e m s ; ~}{ }^{* * *}$ General Health Questionnaire, versión de 12 ítems. 
Tabla 3. Resultados de Validez Convergente. Correlaciones bivariadas entre discapacidad, evaluada con el RDQ y el RDQ-6 y otras variables relevantes

\begin{tabular}{|lcc|}
\hline & RDQ & RDQ-6 \\
Intensidad Dolor (EVA) & $0,235^{*}$ & $0,150^{* *}$ \\
Salud Mental (GHQ-12) & $0,278^{*}$ & $0,178^{* *}$ \\
IMC & 0,015 ns & 0,050 ns \\
Sexo & 0,115 ns & 0,048 ns \\
\hline
\end{tabular}

${ }^{*} p<, 01 ;{ }^{* *} p<, 05 ;$ ns $=$ no significativo.

vulnerabilidad a presentar cuadros de depresión y ansiedad (GHQ-12) y con intensidad del dolor (EVA). Aunque la versión breve del instrumento correlacionó menos significativamente con el GHQ-12 que la tradicional. Por otra parte, ambas versiones no se correlacionaron con el índice de masa corporal, ni con sexo (Tabla 3 ).

\section{Validez de constructo}

Los análisis permiten identificar un factor, tanto en la versión de 24 ítems como en la de 6 ítems del RDQ:

-RDQ. Previo al análisis con el método de Maximun Likehood, se analizó la matriz de correlaciones entre ítems, observándose que la factorización era adecuada $(\mathrm{KMO}=0,793$ y Prueba de Esfericidad de Bartlett $=811,664, \mathrm{gl}=276, \mathrm{p}=$ $0,00)$. Al correr el método de extracción se extrajo un sólo factor, ya que este tenía un eigenvalue superior al resto y era consistente con el Scree Plot observado. Todos los ítems mostraron una carga factorial $>0,25$, excepto por los ítems $1,2,20 \mathrm{y}$ 22. De todos modos se optó por conservar estos ítems con menor carga, ya que su extracción no mejoraba el Alpha de Cronbach de la escala y su peso factorial no era extremamente bajo. Este único componente explicó 19,95\% de la varianza.

-RDQ versión abreviada de 6 ítems. La matriz de correlaciones permitió realizar los análisis planificados (KMO $\geq 0,817$ y Prueba de Esfericidad de Bartlett $\geq 270,007, \mathrm{gl}=15, \mathrm{p}=0,00$ ). Mediante el método de extracción de Maximun Likehood se extrajo un sólo factor para el instrumento abreviado. Sólo un componente presentaba un eigenvalue marcadamente superior, lo que además se evidenciaba en el quiebre de la recta del Scree Plot de los componentes. Este único componente explicó el $30,09 \%$ de la varianza. Las cargas factoriales de los ítems fueron todas $>0,40$.

\section{Discusión}

Los análisis estadísticos realizados muestran que la versión chilena del RDQ, así como una versión abreviada de 6 ítems, tiene características psicométricas solventes al ser aplicado en una muestra chilena de personas con dolor lumbar agudo. Los hallazgos observados indican que ambos cuestionarios son comprensibles, válidos y confiables. En cuanto a la consistencia interna, el Alfa de Cronbach fue para ambas versiones sobre el 0,70 recomendado $^{18}$, lo que da cuenta de una adecuada consistencia interna del cuestionario en ambas versiones. Por otra parte, las correlaciones de la versión abreviada con otras variables del estudio, indican que esta versión se comporta de forma similar a la de 24 ítems. De este modo, consideramos que esta escala breve es un aporte del presente trabajo a la evaluación de la discapacidad, especialmente para el uso clínico.

Los resultados de este estudio en cuanto a la confiabilidad del instrumento original, son consistentes con los estudios de validación del RDQ en diferentes países. Para la versión española, se reportó que el Alfa de Cronbach fue de $0,838^{6}$, muy similar al registrado en el presente estudio, que fue de 0,81 . Con respecto a los puntajes observados con la versión de 6 ítems, estos se consideran aceptables y de acuerdo a la revisión bibliográfica realizada, no existen estudios que hayan realizado análisis con este número de ítems, que permitan un análisis comparativo. La escala de 11 ítems ha mostrado una confiabilidad de 0,88 .

La relación positiva y significativa entre discapacidad y dolor muestra la validez convergente de ambos instrumentos, las versiones del CRM de 24 ítems y de 6 ítems. La magnitud de esta correlación entre dolor y discapacidad en nuestro trabajo es menor que la reportada en trabajos previos, como en la validación tunesina ${ }^{5}$, coreana ${ }^{11}$, así como también en el trabajo original de Roland y Morris $^{14}$. A diferencia de estos trabajos, nuestra muestra no estaba constituida por personas con dolor crónico en el momento de la evaluación, lo que puede explicar la menor relación observada entre discapacidad y dolor en nuestros pacientes. 
La mayor relación entre dolor y discapacidad en pacientes crónicos que en agudos, ha sido previamente reportada ${ }^{19}$.

El estudio de validez convergente muestra que la escala de 6 ítems en general se relaciona con otras variables de la misma forma que la de 24 ítems. Sin embargo, la escala breve, aunque se correlacionó significativamente con síntomas salud mental, lo hizo en menor medida que la original. De esto se puede inferir que la versión de 6 ítems no incorpora los aspectos de salud mental asociados a la discapacidad, de la misma forma que lo hace la de 24 ítems. Esto no es sorprendente en la medida en que no se incluyeron en aquella versión ítems que evalúan directamente aspectos psicológicos, como "Está más irritable y de peor ánimo con las demás personas".

En cuanto a la validez de constructo, el análisis factorial realizado mostró una estructura unidimensional del RDQ. Esto es consistente con los hallazgos de las validaciones gujarati ${ }^{9}$ y japonesa ${ }^{10}$ del instrumento original, quienes también observaron un solo factor. Del mismo modo la versión de 6 ítems mostró un solo factor. Por lo que se considera que la versión de 6 ítems es válida y conserva la estructura del instrumento original.

Respecto a las recomendaciones de uso de esta nueva versión abreviada, se ha establecido que este instrumento permite medir la discapacidad en forma confiable y valida. Sin embargo, se debe tener en cuenta que no parece incorporar la evaluación de los aspectos psicológicos de la discapacidad de la misma forma que la versión original. Además, ninguna de las dos versiones cuenta con un puntaje de corte, por lo que se recomienda usar el RDQ en forma complementaria con observaciones clínicas.

Agradecimientos: A Proyecto VRI-UC a EG. A las psicólogas M. Rodríguez y D. Saenz, quienes contribuyeron con la recolección de los datos y Esperanza Muñoz, quien colaboró en el manuscrito.

\section{Anexo 1. Versión Chilena del RDQ para medir discapacidad}

A continuación hay algunas frases usadas por las personas para describir lo que sienten cuando tienen dolor de espalda. Sólo marque sí cuando esté seguro que esa frase describe lo que usted siente hoy

\begin{tabular}{|c|l|c|c|}
\hline \multicolumn{2}{|l|}{ Debido a su dolor de espalda: } & & sí \\
\hline 1. & Se queda en casa la mayor parte del tiempo. & sí & NO \\
\hline 2. & Se cambia de posición frecuentemente, para ponerse más cómodo. & sí & NO \\
\hline 3. & Camina más lentamente que lo habitual. & sí & NO \\
\hline 4. & Ya no hace ninguno de los trabajos que típicamente hacía en la casa. & sí & NO \\
\hline 5. & Se apoya en la baranda u otra cosa para subir las escaleras. & sí & NO \\
\hline 6. & Se tiende más a menudo durante el día para descansar. & sí & NO \\
\hline 7. & Tiene que afirmarse de algo para levantarse de una silla. & sí & NO \\
\hline 8. & Trata de que otras personas hagan cosas por usted. & sí & NO \\
\hline 9. & Se viste solo, pero lo hace muy lentamente. & sí & NO \\
\hline 10. & Sólo está de pie por períodos corto de tiempo. & sí & NO \\
\hline 11. & Trata de no agacharse o arrodillarse. & sí & NO \\
\hline 12. & Encuentra difícil levantarse de una silla. & sí & NO \\
\hline 13. & La espalda le duele casi todo el tiempo. & sí & NO \\
\hline 14. & Encuentra difícil darse vuelta en la cama. & sí & NO \\
\hline 15. & Su apetito no es muy bueno. & sí & NO \\
\hline 16. & Le cuesta colocarse los calcetines o las medias. & NO \\
\hline 17. & Sólo camina distancias cortas. & Sí & \\
\hline
\end{tabular}


Validación Cuestionario Discapacidad Roland-Morris - E. Guic et al

\begin{tabular}{|c|l|c|c|}
\hline 18. & Duerme peor que de costumbre. & sí & NO \\
\hline 19. & Necesita ayuda de alguien para vestirse. & sí & NO \\
\hline 20. & Pasa sentado la mayor parte del día. & sí & NO \\
\hline 21. & Evita hacer trabajos pesados en la casa. & sí & NO \\
\hline 22. & Está más irritable y de peor ánimo con las demás personas. & sí & NO \\
\hline 23. & Sube escalas más lentamente que lo habitual. & sí & NO \\
\hline 24. & Permanece en cama la mayor parte del tiempo. & sí & NO \\
\hline
\end{tabular}

Escala RDQ versión abreviada de 6 ítems

\begin{tabular}{|c|c|c|c|}
\hline \multicolumn{2}{|c|}{ Debido a su dolor de espalda: } & & \\
\hline 1. & Camina más lentamente que lo habitual. & sí & NO \\
\hline 2. & Trata de que otras personas hagan cosas por usted. & sí & NO \\
\hline 3. & Sólo está de pie por períodos corto de tiempo. & sí & NO \\
\hline 4. & Trata de no agacharse o arrodillarse. & sí & NO \\
\hline 5. & Sólo camina distancias cortas. & sí & NO \\
\hline 6. & Evita hacer trabajos pesados en la casa. & sí & NO \\
\hline
\end{tabular}

\section{Referencias}

1. Ministerio de Salud. Encuesta Nacional de Salud, Chile 2010. Disponible en: http://www.minsal.cl/portal/url/ item/bcb03d7bc28b64dfe040010165012d23.pdf [Consultado el 10 de junio de 2013].

2. Ministerio de Salud. II Encuesta de Calidad de Vida y Salud, Chile 2006. Disponible en: http://epi.minsal.cl/ epi/html/sdesalud/calidaddevida2006/.pdf [Consultado el 10 de junio de 2013].

3. Correa G. Dolor crónico y trauma músculo esquelético: discapacidad e impacto económico. Rev Iberoam del Dolor 2007; 4: 10-8.

4. Exner V, Keel P. Erfassung der Behinderung bei Patienten mit chronischen Rückenschmerzen. Validierung einer deutschen version des Roland \& Morris disability questionnaire sowie verschiedener numerischer Ratingskalen. Schmerz 2000; 14: 392-400.

5. Bejia I, Younes M, Kamel BS, Letaief M, Touzi M, Soltani M, et al. Validation of the Tunisian version of the Roland-Morris Questionnaire. Eur Spine J 2005; 14: 171-4.

6. Kovacs F, Llobera J, Gil De Real M, Abraira V, Gestoso M, Fernández C. Validation of the Spanish version of the Roland Morris questionnaire. Spine 2002; 27: 538-42.

7. Nusbaum L, Natour J, Ferraz MB, Goldenberg J. Translation, adaptation and validation of the Roland-Morris questionnaire-Brazil Roland-Morris. Braz J Med Biol
Res 2001; 34: 203-10.

8. Honlei Y, Xiran J, Xianzhao W, Ziqiang C, Xinhui W, Xiaodong Z. Reliability and validity of simplified Chinese version of Roland-Morris Questionnaire in evaluating rural and urban patients witk low back pain. PLoS ONE 2012; 7 (1): e30807. Disponible en: doi: 10.1371/journal. pone.0030807 [Consultado el 1 de julio de 2013].

9. Nambi SG. Reliability, validity, sensivity and specificity of Gujarati version of the Roland-Morris Disability Questionnaire. Journal of Back and Musculoskeletal Rehabilitation 2013; 20: 149-53.

10. Suzukamo Y, Fukuhara S, Kikuchi S, Konno S, Roland $\mathrm{M}$, Iwamoto $\mathrm{Y}$, et al. Validation of the Japanese version of the Roland-Morris Disability Questionnaire. J Ohtop Sci 2003; 8: 543-8.

11. Slee JS, Lee DH, Suh KT, Kim JI, Lim JM, Goh TS. Validation of the Korean version of the Roland-Morris Disability Questionnaire. Eur Spine J (2011) 20: 2115-9.

12. Stroud MW, Mcknight PE, Jensen MP. Assessment of self-reported physical activity in patients with chronic pain: Development of an abbreviated Roland-Morris Disability Scale. The Journal of Pain 2004; 5: 257-63.

13. Macedo L, Maher CG, Latimer J, Hancock MJ, Machado LAC, Mcauley JH. Responsiveness of the 24-,18 and 11 item versions of the Roland-Morris Disability Questionnaire. Eur Spine J 2011; 20: 458-63.

14. Roland M, Morris R. A study of the natural history of back pain Part I: development of a reliable and sensitive 
measure of disability in low-back pain. Spine 1983; 8: 141-4.

15. Scott J, Huskisson EC. Graphic representation of pain. Pain 1976; 2: 175-84.

16. Goldberg D, Williams P. En: Windsory Berkshire Ed. A user's guide to the General Health Questionnaire. NFER-NELSON, 1988.

17. Trucco M, Valenzuela P. Una batería de cuestionarios para el estudio de estrés ocupacional. Rev Chil NeuroPsiquiat 1998; 36: 159-65.

18. Nunnally JC, Berstein IH, Psychometric Theory (3rd Ed) New York: McGraw Hill, 1994. Grotle M, Brox JI, Vollestad NK. Cross-cultural adaptation of the Norwegian version of the Roland-Morris Disability Questionnaire and the Oswestry Disability Index. J Rehab Med 2003; 35: 241-7. 